\title{
Asymmetry measurements at LEP/SLC revisited
}

\author{
Tariq AZIZ \\ Tata Institute of Fundamental Research, Bombay 400005, India
}

\begin{abstract}
We examine the fermion asymmetry measurements at LEP and SLC leading to ef-

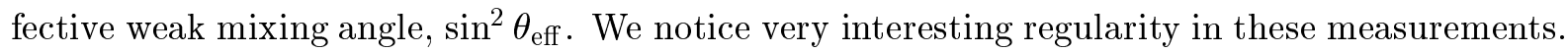
All asymmetry measurements fall in two classes. Class A measurements where hadronisation effects are not relevant for the final result and class B measurements where hadronisation effects can not be avoided and can only be corrected with whatever understanding of these phenomena we have. In each of these classes there is excellent agreement between LEP and SLC results. However the two classes are distinctly apart by more than $3 \sigma$. We suggest that for precision test of the standard model the class A measurements should be preferred.
\end{abstract}

During last few years the standard model has been subjected to very tight precision tests through a variety of measurements at LEP and SLC on one hand and direct confirmation and further tests by another set of measurements at Tevatron on the other $[1,2,3]$. An extremely important measurement at LEP/SLC has been the measurement of the effective weak mixing angle parameter, $\sin ^{2} \theta_{\text {eff }}{ }^{1}$. Whereas in the case of LEP, production of $\mathrm{Z}$ bosons takes place using unpolarised electron/positron beam, in the case of SLC it happens using almost $80 \%$ polarised electron beam. For measuring $\sin ^{2} \theta_{\text {eff }}$ the four LEP experiments ALEPH, DELPHI, L3 and OPAL measure the forward-backward asymmetries for leptons and quarks produced in the $\mathrm{Z}$ decays. They also measure the $\tau$ polarisation asymmetry using $\tau$ decays dominantly in the hadronic channel. On the other hand SLD experiment at SLC measures the polarised asymmetry using the Z production cross-section from left polarised and right polarised beams. Thus the two environments are fairly complementary and help in improving the significance of these measurements even further for reasons of systematics. Taking together all the measurements at LEP and SLC one arrives at an impressive value of $\sin ^{2} \theta_{\text {eff }}=0.23157 \pm 0.00023$ [1]. Since the statistical significance of data in each channel of measurement has reached to fairly high level, it is time to look into some more details of these measurements as various channels have their own systematics. The aim of this paper is to address this issue.

A plot of all the measurements of $\sin ^{2} \theta_{\text {eff }}$ is shown in figure 1 . These measurements

${ }^{1}$ The relationship between $\mathrm{M}_{\mathrm{Z}}$ and $\sin ^{2} \theta_{\text {eff }}$ is given as [4] :

$$
\mathrm{M}_{\mathrm{Z}}^{2} \cos ^{2} \theta_{\mathrm{eff}} \sin ^{2} \theta_{\mathrm{eff}}=\frac{\pi}{\sqrt{2} \mathrm{G}_{\mathrm{F}}} \frac{\alpha}{1-\Delta \mathrm{r}_{\mathrm{eff}}}=\frac{\pi \alpha_{\mathrm{eff}}\left(\mathrm{M}_{\mathrm{Z}}\right)}{\sqrt{2} \mathrm{G}_{\mathrm{F}}}
$$

Where,

$$
\Delta \mathrm{r}_{\mathrm{eff}}=\Delta \alpha+\Delta \mathrm{r}_{\mathrm{W}}
$$

Here $\Delta \alpha$ represents the effect due to running of QED coupling, $\alpha$, in going from low energy to $\mathrm{Z}$ mass scale and $\Delta \mathrm{r}_{\mathrm{W}}$ is the effective weak radiative correction mainly due to top and Higgs in the $\mathrm{Z}$ propagator and some other non leading effects. From now on if the mass scale is not specified, $\alpha$ refers to $\alpha\left(\mathrm{m}_{\mathrm{e}}\right)$. The best estimate of $\alpha$ at $\mathrm{Z}$ mass scale is $\alpha\left(\mathrm{M}_{\mathrm{Z}}\right)=1 /(128.896 \pm 0.090)$ [5]. 
are almost clustered around two values of $\sin ^{2} \theta_{\text {eff }}$. There is the class $\mathrm{A}$ measurements where hadronisation effects are not relevant in determining the sytematics and these are indicated by a label (A). In this class are the measurements of $\mathrm{A}_{\mathrm{FB}}$ of leptons $\mathrm{e} / \mu / \tau$ measured by all experiments at LEP and the left-right polarisation asymmetry $A_{\mathrm{LR}}$ measured by SLD at SLC. There are the other class B measurements where hadronisation effects can not be avoided in determining the systematics. In this class are the measurements of $\mathrm{A}_{\mathrm{FB}}$ of quarks and also the polarisation asymmeties $\mathrm{A}_{\mathrm{e}}$ and $\mathrm{A}_{\tau}$ using $\tau$ decays in the hadronic channel performed by all the experiments at LEP. These are all indicated by another label (B). This trend was noticed earlier [7], when the data were still statistically poor and it was stated that $\sin ^{2} \theta_{\text {eff }}$ determined from asymmetries free from hadronisation effects tend to be smaller than those where such effects are important. It was also stressed that these results should be watched with improvement in precision. Thus as seen in figure 1,

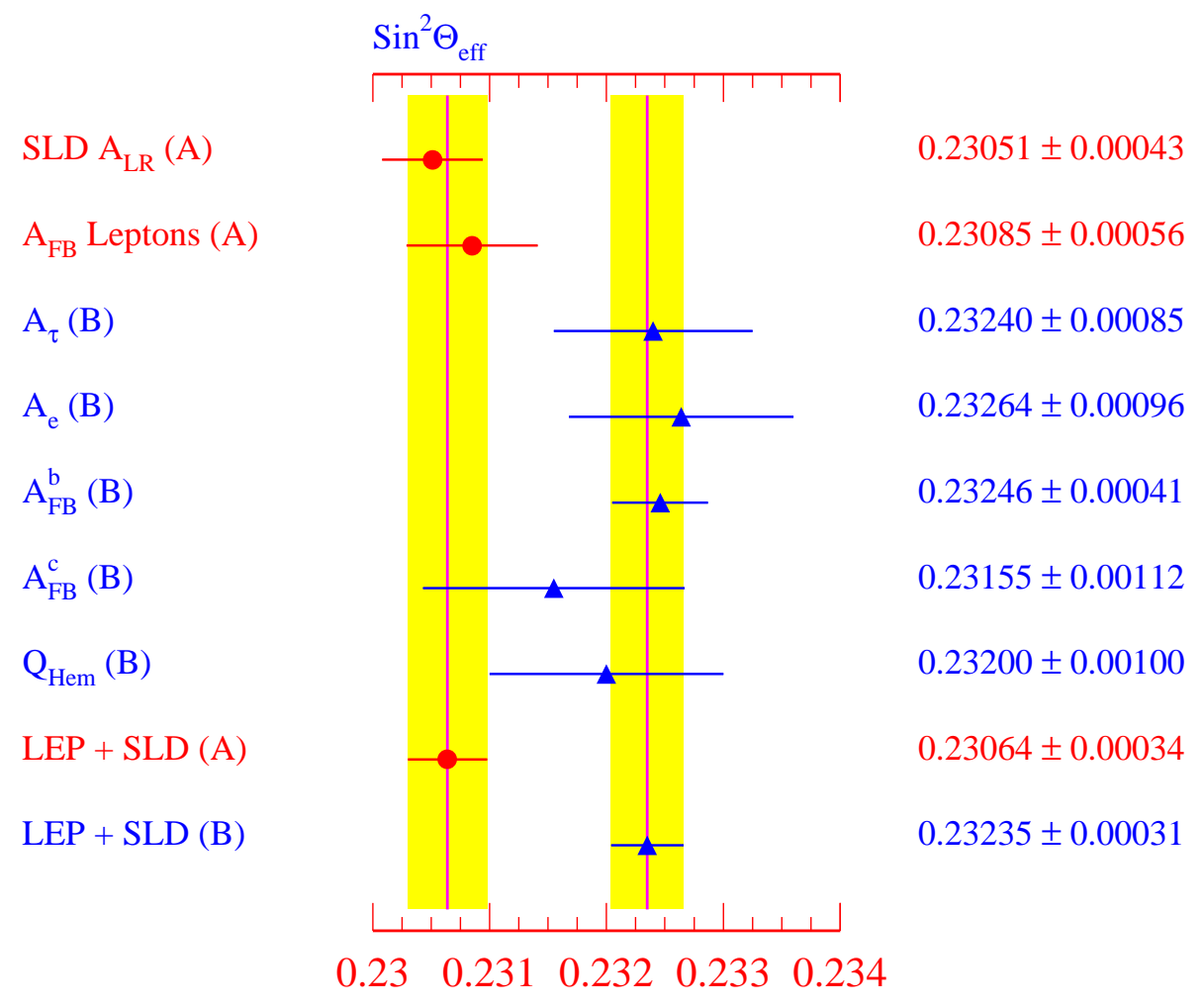

Figure 1: $\sin ^{2} \theta_{\text {eff }}$ measurements using different methods

there is significant difference between the two classes of measurements and the difference is at the level of $3.7 \sigma$. This is the situation inspite of our best efforts in understanding the dilution of asymmetries due to hadronisation effects and the detector modelling of these effects and the reconstruction of jet/hadron four vectors and the flow of charge in the appropriate hemispheres as the situation demands, apart from the effects of missing neutrinos in the case of heavy quarks. Thus there is a need to probe this matter further in as much a model independent way as possible. Fortunately given the LEP and SLC environments where one provides unpolarised beams and the other polarised, some effort can be made. For a general case of polarised or unpolarised beam the produced fermion angular distribution in $\mathrm{Z}$ decay with respect to the beam fermion direction is given by

$$
\frac{\mathrm{d} \sigma}{\mathrm{d} \cos \theta_{\mathrm{f}}} \propto\left(1-\mathrm{A}_{\mathrm{e}} \mathrm{P}_{\mathrm{e}}\right)\left(1+\cos ^{2} \theta_{\mathrm{f}}\right)+2\left(\mathrm{~A}_{\mathrm{e}}-\mathrm{P}_{\mathrm{e}}\right) \mathrm{A}_{\mathrm{f}} \cos \theta_{\mathrm{f}}
$$

where $\mathrm{P}_{\mathrm{e}}$ is the beam polarisation and

$$
\mathrm{A}_{\mathrm{i}}=\frac{2 \mathrm{a}_{\mathrm{i}} \mathrm{v}_{\mathrm{i}}}{\mathrm{a}_{\mathrm{i}}^{2}+\mathrm{v}_{\mathrm{i}}^{2}}=\frac{2\left(1-4\left|\mathrm{Q}_{\mathrm{i}}\right| \sin ^{2} \theta_{\mathrm{eff}}\right)}{1+\left(1-4\left|\mathrm{Q}_{\mathrm{i}}\right| \sin ^{2} \theta_{\mathrm{eff}}\right)^{2}}
$$


where $a_{i}$ and $v_{i}$ are axial and vector couplings for a fermion $i$ with charge $Q_{i}$. Thus for the case of unpolarised beam where $\mathrm{P}_{\mathrm{e}}=0$, the forward-backward asymmetry in $\mathrm{Z} \rightarrow \mathrm{ff}$ is given by

$$
\mathrm{A}_{\mathrm{FB}}^{\mathrm{f}}=\frac{\sigma_{\mathrm{F}}-\sigma_{\mathrm{B}}}{\sigma_{\mathrm{F}}+\sigma_{\mathrm{B}}}=\frac{3}{4} \mathrm{~A}_{\mathrm{e}} \mathrm{A}_{\mathrm{f}}
$$

For the case of polarised beam, $\mathrm{P}_{\mathrm{e}} \neq 0$ like the situation at SLC, the polarised forwardbackward asymmetry is given by

$$
\tilde{\mathrm{A}}_{\mathrm{FB}}^{\mathrm{f}}=\frac{\left(\sigma_{\mathrm{F}}^{\mathrm{L}}-\sigma_{\mathrm{B}}^{\mathrm{L}}\right)+\left(\sigma_{\mathrm{F}}^{\mathrm{R}}-\sigma_{\mathrm{B}}^{\mathrm{R}}\right)}{\left(\sigma_{\mathrm{F}}^{\mathrm{L}}-\sigma_{\mathrm{B}}^{\mathrm{L}}\right)+\left(\sigma_{\mathrm{F}}^{\mathrm{R}}-\sigma_{\mathrm{B}}^{\mathrm{R}}\right)}=\frac{3}{4}\left|\mathrm{P}_{\mathrm{e}}\right| \mathrm{A}_{\mathrm{f}}
$$

Where $\sigma$ 's refer to forward/backward cross-sections or simply the number of events. Thus one immediately notices that in this case there is no dependence on $A_{e}$. To summarise the LEP experiments measure the product $A_{e} \cdot A_{f}$ whereas SLD measures $A_{f}$ from quark asymmetries.

The four LEP experiments ALEPH, DELPHI, L3 and OPAL [1] have measured the b quark asymmetry leading to a combined value $\mathrm{A}_{\mathrm{FB}}^{\mathrm{b}}=0.0979 \pm 0.0023$ after applying all necessary corrections like QCD, QED and $\gamma$ exchange etc. Using this value of $\mathrm{A}_{\mathrm{FB}}^{\mathrm{b}}$ one arrives at $\sin ^{2} \theta_{\text {eff }}=0.23246 \pm 0.00041$. This is more than $2 \sigma$ away from its value $\sin ^{2} \theta_{\text {eff }}=0.23085 \pm 0.00056$ using measured $\mathrm{A}_{\mathrm{FB}}^{\ell}$ at LEP. One can also estimate the value of $A_{b}$ using the expressions mentioned above and the value of $A_{e}$ extracted from the measured $\mathrm{A}_{\mathrm{FB}}^{\ell}$ as this is a measure of $\mathrm{A}_{\mathrm{e}}^{2}$ assuming lepton universality. Restricting only to lepton forward-backward asymmetries at LEP, $\mathrm{A}_{\mathrm{e}}=0.1523 \pm 0.0044$ and thus using the measured value of $\mathrm{A}_{\mathrm{FB}}^{\mathrm{b}}$ one arrives at a value of

$$
\mathrm{A}_{\mathrm{b}(\mathrm{LEP})}=0.857 \pm 0.032
$$

which is more than $2 \sigma$ below its standard model value $\mathrm{A}_{\mathrm{b}(\mathrm{SM})} \simeq 0.935$. Since $\mathrm{SLD}$ measurement of $\widetilde{A}_{\mathrm{FB}}^{\mathrm{b}}$ is independent of $\mathrm{A}_{\mathrm{e}}$, it provides the direct measurement of $\mathrm{A}_{\mathrm{b}}$. The measured value reported by SLD [6] is

$$
\mathrm{A}_{\mathrm{b}(\mathrm{SLD})}=0.863 \pm 0.049
$$

This is in excellent agreement with $\mathrm{A}_{\mathrm{b}(\mathrm{LEP})}$ and an average of the two can be representative of $\left\langle\mathrm{A}_{\mathrm{b}}\right\rangle=0.859 \pm 0.0029$. This is $2.6 \sigma$ below its $\mathrm{SM}$ value.

The nice agreement between LEP and SLD coming from completely different environment of measurement and procedures of analysis makes it difficult to call such agreement mere chance coincidence. In contrast to the measurements, the expected value $\mathrm{A}_{\mathrm{b}(\mathrm{SM})} \simeq 0.935$ is almost independent of $\sin ^{2} \theta_{\text {eff }}$. This is a general situation whenever the vector coupling is substantial (this is the case with quarks, particularly the down type). Thus even the vertex corrections are not relevant for the case of $A_{b}$ and $A_{F B}^{b}$ [8]. In fact any additional correction from any physics consideration can not change the situation substantially unless some drastic changes are introduced in the basic vector and axial vector couplings. In other words the measurement of $A_{b}$ is a good reference point to understand the systematic effects for the measurements mentioned above. An alternative argument can equally well be advanced by using the measured values of $A_{e}$ and $A_{b}$ from SLD to extract the effective value of $b$ quark forward-backward asymmetry similar to LEP and hence the corresponding $\sin ^{2} \theta_{\text {eff }}$ in complete agreement with that obtained at LEP from $\mathrm{A}_{\mathrm{FB}}^{\mathrm{b}}$. Thus whichever way we compare the results at LEP and SLC, there is good agreement and the conclusion 
remains unchanged. This essentially points towards the dilution of asymmetries whenever hadronisation effects become relevant. The dilution appears to be more than what seems to be understood from our present knowledge of hadronisation effects and QCD corrections already implemented in all possible details. The understanding of hadronisation process and subsequent consequences on the quality of b quark direction and the charge assignment are common to all experiments at LEP and SLC and no wonder that they agree so well.

We suggest that either such measurements, where hadronisation effects can not be avoided, are kept aside for precision tests of SM or efforts should be made to minimise them by designing suitable variables where they can be mostly cancelled. Recently the SLD collboration [9] has measured quark charge asymmetries where some combinations lead to cancellation of hadronisation effects. They have measured polarised and unpolarised forward-backward charge asymmeries as

$$
\begin{aligned}
& <\tilde{\mathrm{Q}}_{\mathrm{FB}}>=<\mathrm{Q}_{\mathrm{FB}}^{\mathrm{L}}>\mathrm{f}_{\mathrm{L}}-<\mathrm{Q}_{\mathrm{FB}}^{\mathrm{R}}>\mathrm{f}_{\mathrm{R}} \\
& <\mathrm{Q}_{\mathrm{FB}}>=<\mathrm{Q}_{\mathrm{FB}}^{\mathrm{L}}>\mathrm{f}_{\mathrm{L}}+<\mathrm{Q}_{\mathrm{FB}}^{\mathrm{R}}>\mathrm{f}_{\mathrm{R}}
\end{aligned}
$$

Where $f_{L}$ and $f_{R}$ are the fraction of left- and right-handed events. The ratio of the two quantities above leads to [9]

$$
\mathrm{A}_{\mathrm{Q}}^{\mathrm{obs}}=\frac{<\mathrm{Q}_{\mathrm{FB}}>}{<\tilde{\mathrm{Q}}_{\mathrm{FB}}>}=\frac{\mathrm{A}_{\mathrm{e}}}{\left|\mathrm{P}_{\mathrm{e}}\right|}
$$

where the uncertainties in the detector acceptance, charge assignment and the dilution factors cancel out. This measurement leads to $\sin ^{2} \theta_{\text {eff }}=0.2297 \pm 0.0052 \pm 0.0018$. Our point of view that asymmetry measurements where hadronisation effects can be ignored/cancelled lead to smaller values of $\sin ^{2} \theta_{\text {eff }}$ is well supported though more needs to be done to improve the significance.

We have put other measurements coming from $\tau$ polarisation at LEP like $\mathrm{A}_{\tau}$ and $\mathrm{A}_{\mathrm{e}}$ from which $\sin ^{2} \theta_{\text {eff }}$ is estimated into class $\mathrm{B}$ where hadronisation effects are relevant. The reason being that for $\tau$ polarisation measurements at LEP, the hadronic final states of $\tau$ decay $(\tau \rightarrow \rho \nu, \rightarrow \pi \nu)$ play important role and understanding energy and angular resolution of the decay products is relevant. Obviously they fit more into the class of measurements where quarks are placed and one can see the excellent agreement in $\sin ^{2} \theta_{\text {eff }}$ values displayed in the figure 1.

Given the status of most of the $\sin ^{2} \theta_{\text {eff }}$ measurements, we can summarise the overall situation on asymmetry measuements. We find that there is excellent agreement between LEP and SLC experiments if the measurements are classified properly. Thus we suggest that all those measurements where hadronisation effects can not be avoided/cancelled should be considered in one class and those which are free from such effects should be considered in another class and the latter should be preferred for obvious reasons. Thus we suggest the best representative value of $\sin ^{2} \theta_{\text {eff }}$ measurements for LEP and SLC is from this class of measurements as $\sin ^{2} \theta_{\text {eff }}(\mathrm{LEP}+\mathrm{SLC})_{\mathrm{A}}=0.23064 \pm 0.00034$.

Further support to our view comes from observing the W boson mass measurements at $\overline{\mathrm{P} P}$ collider at Tevatron over the last few years [3] and recent measurements at LEP [10]. The trend shows that $\mathrm{W}$ mass has been rising with time. The current best average of 


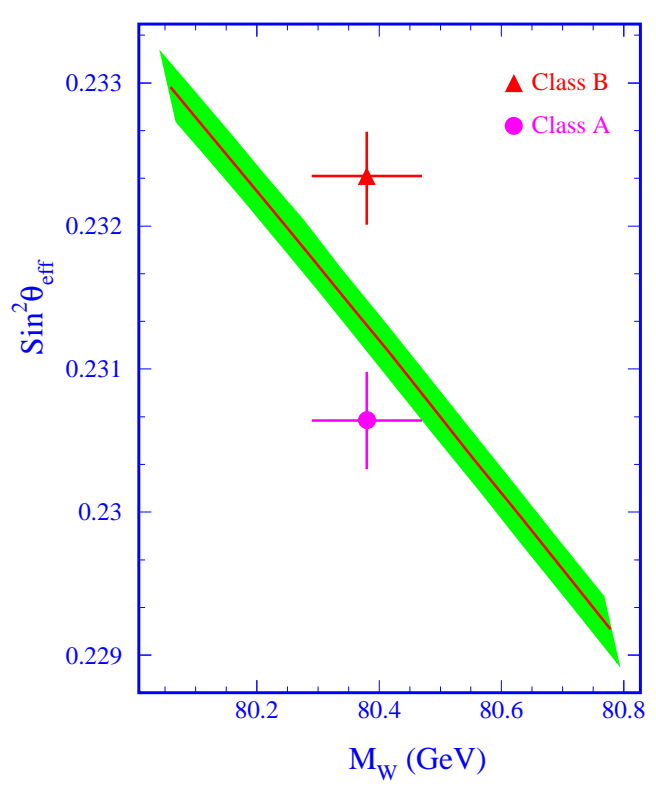

Figure 2: Dependence of $\sin ^{2} \theta_{\text {eff }}$ on $\mathrm{W}$ boson mass. The band around central line corresponds to $\alpha\left(\mathrm{M}_{\mathrm{Z}}\right)$ uncertainty. The data points shown correspond to $\sin ^{2} \theta_{\text {eff }}$ from class $\mathrm{A}$ and B measurements in combination with $\mathrm{W}$ mass from Tevatron and LEP experiments.

these measurements is $\mathrm{M}_{\mathrm{W}}=80.38 \pm 0.09 \mathrm{GeV}$ leading to $\sin ^{2} \theta_{\text {eff }}\left(\mathrm{M}_{\mathrm{W}}\right)=0.2313 \pm 0.0006$ within SM framework. The variation of $\sin ^{2} \theta_{\text {eff }}$ on $\mathrm{W}$ mass within the framework of SM is shown in figure 2 where the two data points correspond to $\sin ^{2} \theta_{\text {eff }}$ for the two classes $\mathrm{A}$ and $\mathrm{B}$ in combination with the measured $\mathrm{W}$ mass. The advantage of this plot is that it does not require any specific Higgs or top quark mass for interpretation and provides a simple internal consistency check of data. Thus if the data is expected to agree with the SM then it should follow the band. In future one would also like an improvement in $\alpha\left(\mathrm{M}_{\mathrm{Z}}\right)$ apart from improvement in $\sin ^{2} \theta_{\text {eff }}$ and $\mathrm{M}_{\mathrm{W}}$. Given the present status of data it is obvious from figure 2 that class A measurements of $\sin ^{2} \theta_{\text {eff }}$ are favoured and any further increase in $\mathrm{W}$ mass will make the agreement better.

In figure 3 we have plotted $\sin ^{2} \theta_{\text {eff }}$ as a function of top quark mass. The data corresponds to $\mathrm{M}_{\mathrm{t}}=175 \pm 6 \mathrm{GeV}$ from CDF and D0 experiments [11] and $\sin ^{2} \theta_{\text {eff }}=0.23064 \pm 0.00034$ from class A measurements. From this figures it is obvious that light Higgs is very much favoured. However, based on direct searches at LEP, a light Higgs $\left(\mathrm{M}_{\mathrm{H}}<70 \mathrm{GeV}\right)$ is nowhere in sight. Thus a desirable option would be a few $\mathrm{GeV}$ increase in top quark mass and/or a small change in $\sin ^{2} \theta_{\text {eff }}$. Given the top mass measurements where hadron calorimetry and understanding jets (energy inside the accepted cone versus left outside)

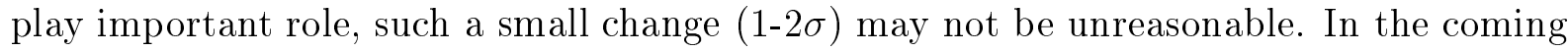
years when Tevatron has plenty of top produced this will become clear.

Finally we would like to add that taking all the electroweak measurements together we see a converging trend of all the measurements such that $\sin ^{2} \theta_{\text {eff }}$ remains close to $\simeq 0.2311$, within the overlapping band shown in figure 3. In the coming years when SLD improves its $\sin ^{2} \theta_{\text {eff }}$ measurements significantly and LEP and Tevatron have improved the W mass measurements further, this will become clear. However seeing figure 3 , one finds that the effects of weak radiative corrections in $\sin ^{2} \theta_{\text {eff }}$ are least visible for such a situation. In fact we end up with a two fold ambiguity because such a value of $\sin ^{2} \theta_{\text {eff }}$ can also be obtained by simply runing $\alpha_{\mathrm{QED}}$ upto $\mathrm{Z}$ mass scale as shown by the overlapping bands. This is 
[4] M. Consoli, W. Hollik and F. Jegerlehner, Z Physics at LEP, CERN report: CERN 89-08, Vol 1., p 7. Eds: G. Altarelli etal.

G. Altarelli, R. Barbieri and S. Jadach, Nucl. Phys. B369, 3 (1992).

[5] S.Eidelmann and F. Jegerlehner, Z. Phys. C67, 585 (1995).

[6] SLD Collaboration, K. Abe etal. SLAC-PUB-7019 (1995); SLAC-PUB-7233 (1996). Presented at ICHEP, Warsaw, Poland (1996).

[7] T. AZIZ, Mod. Phys. Lett. A9, 1857 (1994).

[8] T. AZIZ, Some interesting approximations of $\mathrm{A}_{\mathrm{FB}}$ on $\mathrm{Z}^{0}$ peak, TIFR-EHEP-90/6, November 1996

L3 Collaboration, Phys. Lett. B252, 713 (1990).

[9] SLD Collaboration, K. Abe etal. Phys. Rev. Lett. 78, 17 (1997).

[10] LEP Results on W mass measurements presented at ASPEN Conference, January 1997. Also reported at La Thuile, Italy, March 1997.

[11] CDF Collaboration, Result presented at ICHEP, Warsaw, Poland, July 1996. Also reported at La Thuil, Italy, March 1997.

D0 Collaboration, Result presented at ICHEP, Warsaw, Poland, July 1996. Also reported at La Thuil, Italy, March 1997. 